\title{
ИНГИБИТОРЫ НАТРИЙ-ГЛЮКОЗНОГО КОТРАНСПОРТЕРА СПОСОБНЫ ПОЛОЖИТЕЛЬНО ВЛИЯТЬ НА СОСТОЯНИЕ ГОЛОВНОГО МОЗГА
}

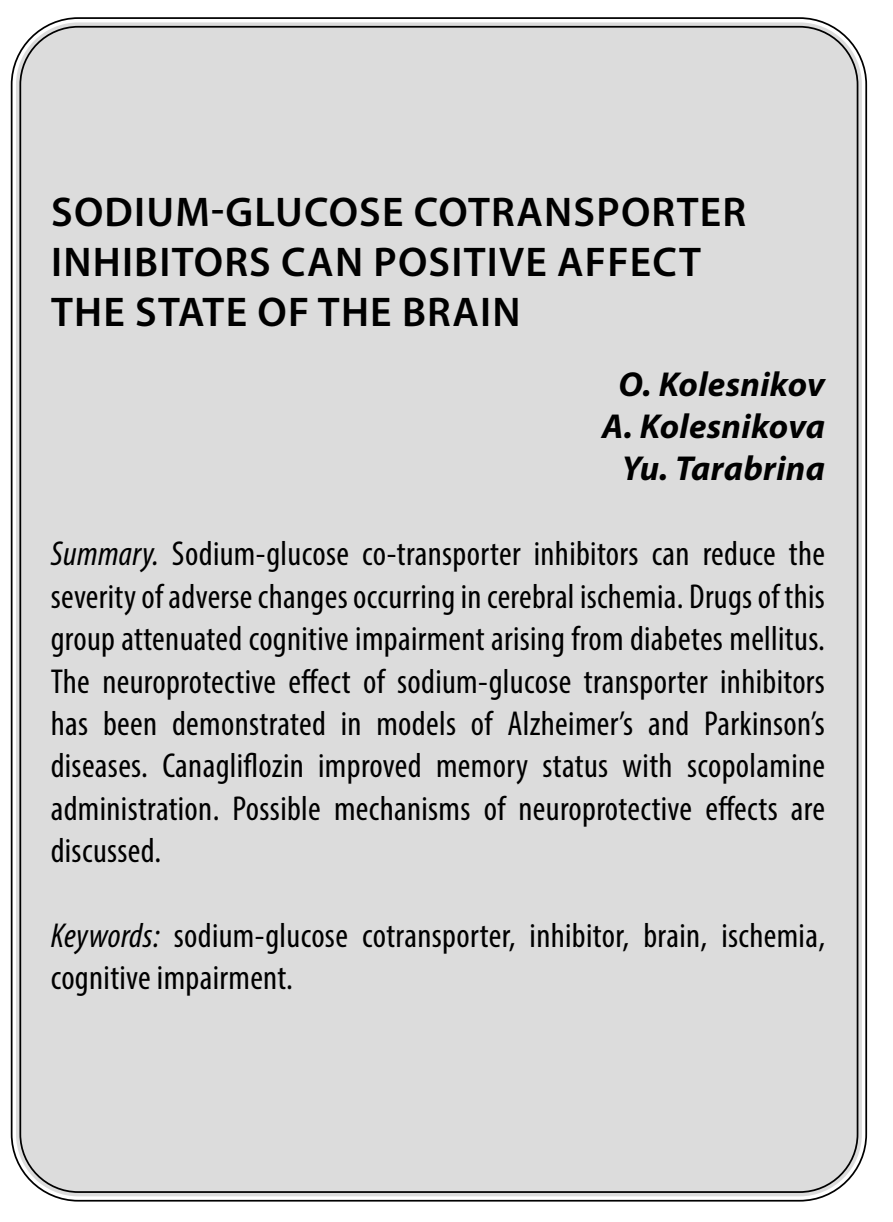

и нгибиторы натрий-глюкозного котранспортера 2 (ИНГК2) были разработаны для лечения сахарного диабета (СД) и сегодня широко применяются в медицинской практике [1]. В процессе клинических исследований было обнаружено, что ИНГК2 кроме сахароснижающего действия обладают также кардиопротекторными и ренопротекторными свойствами. Использование этих препаратов замедляет скорость прогрессирования сердечной недостаточности $(\mathrm{CH})$, снижает количество госпитализаций по поводу $\mathrm{CH}$, coкращает смертность, связанную с сердечно-сосудистой патологией, благоприятно влияет на пациентов с поражением почек $[2,3,4,5]$.

Совет экспертов, проходивший в Москве в 2019 году, констатировал, что можно рассматривать дапаглифлозин (относящийся к ИНГК2) как дополнение к общепри-
Колесников Олег Леонидович

Д.м.н., професссор, ФГБОУ ВО «Южно-Уральский государственный медицинский университет» Минздрава России (2. Челябинск) kaf-biol@mail.ru

Колесникова Алла Алексеевна

К.м.н., дочент, ФГБОУ ВО «Южно-Уральский государственный медицинский университет» Минздрава России (2. Челябинск) olekol@mail.ru

Тарабрина Юлия Олеговна

К.м.н., доцент, ФГБОУ ВО «Южно-Уральский государственный медицинский университет» Минздрава России (г. Челябинск) julikol@mail.ru

Аннотация. Ингибиторы натрий-глюкозного котранспортера способны снижать выраженность неблагоприятных изменений, возникающих при ишемии головного мозга. Препараты этой группы ослабляли когнитивные нарушения, возникающие при сахарном диабете. Нейропротекторное действие ингибиторов натрий-глюкозного транспортера продемонстрировано на моделях болезней Альцгеймера и Паркинсона. Канаглифлозин улучшал состояние памяти при введении скополамина. Обсуждены в0зможные механизмы нейропротективных эффектов.

Ключевые слова: натрий-глюкозный котранспортер, ингибитор, головной мозг, ишемия, когнитивные нарушения.

нятой терапии хронической сердечной недостаточности со сниженной фракцией выброса левого желудочка. Это позволит улучшить прогноз выживаемости пациентов. При этом подана заявка на регистрацию нового показания для применения дапаглифлозина [6].

Настоящая статья посвящена новой, слабо изученной способности ингибиторов натрий-глюкозного котранспортера - влиянию на состояние и функционирование головного мозга.

Головной мозг для нормального функционирования расходует большое количество энергии. Энергия в основном обеспечивается за счет метаболизма глюкозы. Поэтому мозг нуждается в постоянном поступлении глюкозы [7, 8]. По данным Mink J.W. и соавт. (1981) мозг потребляет около $20 \%$ всей полученной с пищей глю- 
козы [9]. Указанное обстоятельство требует высокой активности переносчиков глюкозы. В головном мозге функционируют транспортеры глюкозы (GLUTs), натрий-глюкозные котранспортеры (SGLTs) и SWEETs [10]. Экспрессия натрий-глюкозных котранспортеров (НГК) 1 и 2 обнаружена в нейронах различных отделов мозга, а также в эндотелии капилляров мозга [7, 11, 12].

Сначала остановимся на данных о последствиях церебральной ишемии. В работе Yamazaki Ү. и соавт. (2012) использовали мышей линии ddY, которых подвергали 2-часовой окклюзии средней мозговой артерии [13]. Было показано наличие постишемической гипергликемии. При этом внутрибрюшинное введение флоризина (ИНГК 1 и 2) выраженно, статистически значимо и дозозависимо подавляло повышение глюкозы и ишемическое повреждение нейронов. Если же флоризин вводили внутрь желудочков мозга, то отмечали только достоверное уменьшение неблагоприятных изменений в нейронах без снижения уровня глюкозы [13]. В дальнейшем исследователи после окклюзии артерий в желудочки мозга вводили глюкозу, что приводило к заметному отягощению отрицательных последствий ишемии. Если же параллельно использовали глюкозу и флоризин, отягощения последствий ишемии не наблюдалось [13].

Harada S. и соавт. (2013) использовали модель 30-минутной двусторонней окклюзии сонной артерии у мышей [14]. На третий день после окклюзии мышам вводили флоризин (одной группе интраперитонеально, второй группе - в желудочки мозга). Использование флоризина значительно и достоверно подавляло выраженность ишемического повреждения нейронов. Также проводили анализ походки экспериментальных животных. Было обнаружено, что только внутрижелудочковое введение флоризина подавляло спастичность у мышей [14].

В одной из работ изучали культуру первичных кортикальных нейронов, полученных от плодов мышей ddY. Инкубация клеток в присутствии глюкозы в высокой концентрации индуцировала увеличение концентрации $\mathrm{Na}+$ в цитоплазме. Это явление было подавлено ингибитором НГК 1 и 2 флоризином. Специфичный для НГК приток $\mathrm{Na}+\mathrm{B}$ цитоплазму клетки был индуцирован с помощью обработки культуры а-метил-D-глюкопиранозидом. В результате значительно снизилась выживаемость нейронов и увеличилась гибель клеток, вызванная перекисью водорода. Флоризин достоверно ослаблял эти эффекты [15]. Ученые предположили, что интенсивное поступление $\mathrm{Na}+$ в цитоплазму нейронов с помощью НГК может усугубить развитие ишемического повреждения нейронов головного мозга [15].
В условиях ишемии/реперфузии эмпаглифлозин ограничивал объем инфаркта [16]. Wiciński M. и соавт. (2020) вызывали у крыс ишемию мозга путем окклюзии сонных артерий. Эмпаглифлозин ослаблял неврологические дефекты у животных. При изучении механизмов этого позитивного эффекта исследователи обнаружили, что ИНГК2 вызывал рост уровня индуцированного гипоксией фактора 1 a (HIF-1a) и повышенную экспрессию сосудистого эндотелиального фактора роста A (VEGF-A) [17]. Известно, что HIF-1 а обеспечивает восстановление кислородного гомеостаза путем активации гликолиза, эритропоэза и ангиогенеза [18]. При повышении уровня HIF-1a также достоверно снижалась экспрессия рецепторов провоспалительных цитокинов ИЛ-6 и ФНО-а [19]. Не меньшее значение в борьбе с последствиями ишемии имеет и VEGF-A. Сосудистые эндотелиальные факторы роста известны как важные регуляторы ангиогенеза и нейрогенеза [20]. Также можно упомянуть тот факт, что у грызунов вида Heterocephalus glaber обнаружена очень высокая врожденная толерантность к гипоксии. Исследователи полагают, что этот интересный феномен, вероятно, связан с очень высокой экспрессией VEGF-A [21].

Вторая часть статьи посвящена данным о влиянии ИНГК на когнитивные функции. В нейронах головного мозга и клетках глии была обнаружена экспрессия и активность инсулиновых рецепторов. При этом нарушение сигнальной цепи инсулина в головном мозге связано с аномальной функцией нейронов [22]. Резистентность головного мозга к инсулину играет значительную роль в формировании когнитивных дисфункций, в том числе при сахарном диабете 2 типа (СД2) [23, 24]. Инсулинорезистентность головного мозга сопровождается нарушением нейропластичности, активацией воспалительных процессов, дисфункцией митохондрий, уменьшением выраженности дендритных шипов и снижением уровня полученного из мозга нейротрофического фактора (brain derived neurotrophic factor BDNF) $[25,26,27]$.

Пациенты с СД2 имеют высокий риск развития когнитивных нарушений, которые могут варьировать от небольших сдвигов до преддеменции и собственно деменции. Есть предположения, что данный факт связан с комплексом факторов: инсулинорезистентность, нарушение обмена глюкозы, митохондриальная дисфункция, нейровоспаление и сосудистая патология [28, 29]. При наличии сахарного диабета риск развития когнитивных нарушений и деменции в 1.5-2.5 раза выше, чем у людей без диабета [30, 31].

Sa-Nguanmoo P. и соавт. (2017) содержали крыс на диете с высоким содержанием жира. У экспериментальных животных развилась резистентность к инсу- 
лину с ожирением и снижение когнитивных функций. Дапаглифлозин улучшал периферическую чувствительность к инсулину, функции митохондрий головного мозга, снижал апоптоз и предотвращал снижение когнитивных функций. Кроме того, ИНГК2 улучшал синаптическую пластичность гиппокампа [32].

В исследовании Lin B. и соавт. (2014) изучали мышей линии $\mathrm{db} / \mathrm{db}$, которые являются моделью СД2, одна из групп животных получала эмпаглифлозин. Контролем были мыши линии $\mathrm{db} / \mathrm{m}$ (без СД). Для оценки когнитивных способностей применяли водный лабиринт Морриса. Латентность спасения на скрытой платформе была достоверно больше у мышей $\mathrm{db} / \mathrm{db}$, чем у мышей $\mathrm{db} / \mathrm{m}$. При отсутствии скрытой платформы количество пересечений места её типичного расположения у мышей с СД2 было почти в 2.5 раза меньше, чем у здоровых животных. Сделан вывод, что при наличии СД2 происходит значительное ухудшение обучения и памяти. При использовании эмпаглифлозина задержка в обнаружении скрытой платформы статистически значимо сокращалась. Эмпаглифлозин также восстанавливал число пересечений места расположения платформы практически до контрольного уровня. Различия с мышами, не получавшими ИНГК2, были статистически достоверны. Приведенные факты свидетельствуют о коррекции нарушения когнитивных способностей у животных с СД2 [33].

Кроме того, было показано, что при использовании эмпаглифлозина в головном мозге статистически значимо уменьшался уровень супероксида и 8-гидрокси-дезоксигуанозина, который является маркером повреждения ДНК. При этом лечение эмпаглифлозином статистически значимо более чем в 1.5 раза повышало содержание BDNF в головном мозге [33]. BDNF важен не только для развития нервной системы, он также способствует поддержанию гомеостаза в мозге взрослого человека. BDNF необходим для выживания как развивающихся, так и взрослых нейронов [34]. У мышей с частичным нокаутом BDNF наблюдается нарушение выживаемости и функции нейронов стриатума [35]. А снижение уровня церебрального BDNF связано с когнитивными нарушениями [36]. Высказано предположение, что увеличение содержания BDNF, а также ослабление окислительного стресса, по-видимому, обеспечивают предотвращение когнитивных нарушений при СД2 с помощью эмпаглифлозина [33].

Позитивное действие ИНГК было зафиксировано не только в отношении функций мозга при СД2. При изучении мышей, у которых индуцировали болезнь Альцгеймера, зафиксировали нарушение когнитивных способностей, наличие оксидативного стресса, повышенную активность ацетилхолинэстеразы (АХЭ). Так- же обнаружили выраженную инфильтрацию тканей нейтрофилами. При введении флоризина (ингибитора НГК1 и НГК2) когнитивные нарушения, биохимические сдвиги и гистопатологические изменения значительно смягчались [37].

Hierro-Bujalance C. и соавт. (2020) изучали три линии мышей: db/db (модель СД2), APP/PS1 (модель болезни Альцгеймера - БА) и APP/PS1 $\mathrm{xdb} / \mathrm{db}$ (модель одновременно БА и СД2). Для оценки когнитивных способностей применяли водный лабиринт Морриса. Время обнаружения скрытой платформы возрастало у мышей с БА, СД2 и сочетанием БА+СД2, что указывало на наличие когнитивных нарушений. Лечение эмпаглифлозином достоверно сокращало этот показатель. При удалении платформы время нахождения мышей в квадранте, который ранее содержал скрытую платформу, уменьшалось во всех исследуемых группах (БА, СД2 и БА+СД2). Этот факт свидетельствовал об ухудшении памяти. Применение эмпаглифлозина восстанавливало память у мышей с СД2 и сочетанием БА+СД2. Кроме того, у животных с БА, СД2 и сочетанием этих патологий статистически достоверно снижалась плотность нейронов в коре головного мозга. Эмпаглифлозин достоверно увеличивал плотность нейронов во всех исследуемых группах [38]. АХЭ является одной из мишеней при лечении БА. При изучении энергии связи препарат - АХЭ и оценке взаимодействия препаратов с аминокислотами было показано, что такие ИНГК2 как канаглифлозин, дапаглифлозин и сотаглифлозин способны связываться с АХЭ и могут быть использованы как ингибиторы этого фермента [39, 40, 41].

В работе Arab H.Н. и соавт. (2021) у крыс индуцировали формирование болезни Паркинсона с помощью ротенона, часть животных получала в дапаглифлозин течение 3 недель. ИНГК2 ослаблял двигательную дисфункцию и улучшал координацию движений в тестах «открытое поле» и «вращающийся стержень». Также обнаружено уменьшение гистопатологических изменений мозга и увеличение уровня дофамина. Одновременно ИНГК2 заметно снижал окислительный стресс нейронов за счет подавления липопероксидации. Дапаглифлозин подавлял нейровоспаление, снижая уровень фактора некроза опухолей а [42].

При использовании скополамина у крыс обнаружили наличие когнитивной дисфункции и нарушение памяти. При использовании канаглифлозина значительно улучшились показатели, демонстрируемые животными в крестообразном лабиринте и водном лабиринте. Также при использовании ИНГК2 значительно снизилась активность АХЭ и возросли уровни моноаминов. Сделан вывод, что канаглифлозин может улучшить состояние памяти, поврежденной скополамином [43]. 


\section{ЛИТЕРАТУРА}

1. Padhi S. Type II diabetes mellitus: a review on recent drug based therapeutics / S. Padhi, A.K. Nayak, A. Behera// Biomed. Pharmacother. - 2020.— Vol. 131. - Article ID: 110708. D0I: 10.1016/j.biopha.2020.110708.

2. Johansen M.E. The cardiovascular outcomes, heart failure and kidney disease trials tell that the time to use Sodium Glucose Cotransporter 2 inhibitors is now / M.E. Johansen, C. Argyropoulos // Clin. Cardiol._ 2020.—Vol. 43._P. 1376-1387. D0I: 10.1002/clc.23508.

3. Vasquez-Rios G. SGLT2 Inhibitors: Emerging Roles in the Protection Against Cardiovascular and Kidney Disease Among Diabetic Patients / G. Vasquez-Rios, G.N. Nadkarni // Int. J. Nephrol. Renovasc. Dis. — 2020. — Vol. 13. — P. 281-296. D0I: 10.2147/IJNRD.S268811.

4. Cheng J.W.M. Focused Updates: SGLT2 Inhibitors in Patients With Heart Failure and/or Chronic Kidney Disease / J.W.M. Cheng, V. Colucci, J.S. Kalus, S.A. Spinler // Ann. Pharmacother. - 2021. - Vol. 55, N2. - P. 252-260. D01: 10.1177/1060028020934001.

5. O'Meara E. When and How to Use Sodium-Glucose Cotransporter 2 Inhibitors in Patients With Heart Failure With Reduced Ejection Fraction or Chronic Kidney Disease / E. 0'Meara, S. Verma // Can. J. Cardiol. — 2021.—Vol. 37, N4.—P. 669-673. D0l: 10.1016/j.cjca.2021.01.005.

6. Терещенко С.Н. Целесообразность назначения дапаглифлозина для профилактики неблагоприятных исходов хронической сердечной недостаточности у пациентов со сниженной фракцией выброса. Резолюция совета экспертов / С.Н. Терещенко, М.В. Шестакова, Ф.Т. Агеев [и др.] // Российский кардиологический журнал. - 2020. — Том 25, № 5.—C. 114-120. D0I:10.15829/1560-4071-2020-3919.

7. Koepsell H. Glucose transporters in brain in health and disease / H. Koepsell // Pflugers Arch. — 2020. — Vol. 472, N9. — P. 1299-1343. D0I: 10.1007/s00424020-02441-x.

8. Siesjö B.K. Brain energy metabolism and catecholaminergic activity in hypoxia, hypercapnia and ischemia / B.K. Siesjö // J. Neural. Transm. Suppl. — 1978. — Vol. 14.-P. 17-22.

9. Mink J.W. Ratio of central nervous system to body metabolism in vertebrates: its constancy and functional basis / J.W. Mink, R.J. Blumenschine, D.B. Adams // Am.J. Physiol. - 1981.—Vol. 241, N3.—P. R203-R212. D0I: 10.1152/ajpregu.1981.241.3.R203.

10. Chen Li-Q. Transport of sugars / Li-Q. Chen, L.S. Cheung, L. Feng [et al.] // Annu. Rev. Biochem. — 2015.— Vol. 84. — P. 865-894. D0I: 10.1146/annurevbiochem-060614-033904.

11. Enerson B.E. The rat blood-brain barrier transcriptome / B.E. Enerson, L.R. Drewes // J. Cereb. Blood Flow Metab. — 2006. — Vol. 26, N7.—P. 959-973. D0I: 10.1038/sj.jcbfm. 9600249 .

12. Chen J. Quantitative PCR tissue expression profiling of the human SGLT2 gene and related family members / J. Chen, S. Williams, S. Ho [et al.] // Diabetes Ther. - 2010.—Vol. 1, N2.—P. 57-92. D0l: 10.1007/s13300-010-0006-4.

13. Yamazaki Y. Post-ischemic hyperglycemia exacerbates the development of cerebral ischemic neuronal damage through the cerebral sodium-glucose transporter / Y. Yamazaki, S. Harada, S. Tokuyama // Brain Res. — 2012.—Vol. 1489.— P. 113-120. D0I: 10.1016/j.brainres.2012.10.020.

14. Harada S. Neuroprotective effect through the cerebral sodium-glucose transporter on the development of ischemic damage in global ischemia / S. Harada, Y. Yamazaki, H. Nishioka, S. Tokuyama // Brain Res. — 2013.—Vol. 6. - P. 1541. — P. 61-68. D0I: 10.1016/j.brainres.2013.09.041.

15. Yamazaki Y. Sodium transport through the cerebral sodium-glucose transporter exacerbates neuron damage during cerebral ischaemia /Y. Yamazaki, S. Harada, T. Wada [et al.] // J. Pharm. Pharmacol. — 2016. — Vol. 68, N7. — P. 922-931. D0l: 10.1111/jphp.12571.

16. Abdel-Latif R.G. Empagliflozin alleviates neuronal apoptosis induced by cerebral ischemia/reperfusion injury through HIF-1a/VEGF signaling pathway / R.G. Abdel-Latif, R.A. Rifaai, E.F. Amin // Arch. Pharm. Res. - 2020. — Vol. 43, N5. - P. 514-525. D0I: 10.1007/s12272-020-01237-y.

17. Wiciński M. Perspective of SGLT2 Inhibition in Treatment of Conditions Connected to Neuronal Loss: Focus on Alzheimer's Disease and Ischemia-Related Brain Injury / M. Wiciński, E. Wódkiewicz, K. Górski [et al.] // Pharmaceuticals (Basel). — 2020.—Vol. 13, N11.—Article ID: 379. D0l: 10.3390/ph13110379.

18. Carmeliet P. Role of HIF-1alpha in hypoxia-mediated apoptosis, cell proliferation and tumour angiogenesis / P. Carmeliet, Y. Dor, J.M. Herbert [et al.] // Nature. — 1998. - Vol. 394, N6692. — P. 485-490. D01: 10.1038/28867.

19. Xing J. HIF-1a Activation Attenuates IL-6 and TNF-a Pathways in Hippocampus of Rats Following Transient Global Ischemia / J. Xing, J. Lu // Cell Physiol. Biochem. - 2016. - Vol. 39, N2. — P. 511-520. D01: 10.1159/000445643.

20. Thau-Zuchman 0. Vascular endothelial growth factor increases neurogenesis after traumatic brain injury / 0. Thau-Zuchman, E. Shohami, A.G. Alexandrovich, R.R. Leker // J. Cereb. Blood Flow Metab. - 2010.—Vol. 30, N5. - P. 1008-1016. D0l: 10.1038/jcbfm.2009.271.

21. Larson J. No oxygen? No problem! Intrinsic brain tolerance to hypoxia in vertebrates / J. Larson, K.L. Drew, L.P. Folkow [et al.] // J. Exp. Biol. — 2014. — Vol. 217, Pt. 7. - P. 1024-1039. D0l: 10.1242/jeb.085381.

22. Kleinridders A. Insulin action in brain regulates systemic metabolism and brain function /, H.A. Ferris, W. Cai, C.R. Kahn // Diabetes. - 2014. - Vol. 63, N7. P. 2232-2243. D0l: 10.2337/db14-0568.

23. Kullmann S. Brain Insulin Resistance at the Crossroads of Metabolic and Cognitive Disorders in Humans / S. Kullmann, M. Heni, M. Hallschmid [et al.] // Physiol. Rev. - 2016. - Vol. 96, N4. - P. 1169-1209. D0l: 10.1152/physrev.00032.2015.

24. Kim H.-G. Cognitive dysfunctions in individuals with diabetes mellitus / H.-G. Kim // Yeungnam. Univ. J. Med. — 2019.— Vol. 36, N3. — P. 183-191. D0I: 10.12701/yujm.2019.00255

25. Chen Q. Repurposing of Anti-Diabetic Agents as a New Opportunity to Alleviate Cognitive Impairment in Neurodegenerative and Neuropsychiatric Disorders / Q. Chen, T. Cao, N. Li [et al.] // Front. Pharmacol. — 2021._Vol. 12.—Article ID: 667874. D0I: 10.3389/fphar.2021.667874.

26. Biessels G.J.Hippocampal insulin resistance and cognitive dysfunction / G.J. Biessels, L.P. Reagan // Nat. Rev. Neurosci.— 2015.—Vol. 16, N11.—P. 660-671. D0I: 10.1038/nrn4019. 
27. Park H.-S. Exercise Alleviates Cognitive Functions by Enhancing Hippocampal Insulin Signaling and Neuroplasticity in High-Fat Diet-Induced Obesity / H.-S. Park, S.-S. Park, C.-J. Kim. [et al.] // Nutrients. — 2019._- Vol. 11, N7.— Article ID: 1603. D0I: 10.3390/nu11071603.

28. Biessels G.J. Cognitive decline and dementia in diabetes mellitus: mechanisms and clinical implications / G.J. Biessels, F. Despa // Nat. Rev. Endocrinol.2018. — Vol. 14, N10. — P. 591-604. D0l: 10.1038/s41574-018-0048-7.

29. Papazafiropoulou A.K. Diabetes and dementia - the two faces of Janus / A.K. Papazafiropoulou, C. Koros, A. Melidonis, S. Antonopoulos // Arch. Med. Sci. Atheroscler. Dis. - 2020.—Vol. 5.—P. e186-e197. D0I: 10.5114/amsad.2020.97433.

30. Cukierman T. Cognitive decline and dementia in diabetes - systematic overview of prospective observational studies / T. Cukierman, H.C. Gerstein, J.D. Williamson // Diabetologia. — 2005. — Vol. 48, N12.—P. 2460-2469. D01: 10.1007/s00125-005-0023-4.

31. Salas I.H. Diabetes and Alzheimer's Disease: A Link not as Simple as it Seems / I.H. Salas, B. De Strooper // Neurochem. Res. — 2019. — Vol. 44, N6. — P. 12711278. D0I: 10.1007/s11064-018-2690-9.

32. Sa-Nguanmoo P. SGLT2-inhibitor and DPP-4 inhibitor improve brain function via attenuating mitochondrial dysfunction, insulin resistance, inflammation, and apoptosis in HFD-induced obese rats / P. Sa-Nguanmo0, P. Tanajak, S. Kerdphoo [et al.] // Toxicol. Appl. Pharmacol.— 2017.— Vol. 333.— P. 43-50. D0l: 10.1016/j.taap.2017.08.005.

33. Lin B. Glycemic control with empagliflozin, a novel selective SGLT2 inhibitor, ameliorates cardiovascular injury and cognitive dysfunction in obese and type 2 diabetic mice / B. Lin, N. Koibuchi, Y. Hasegawa [et al.] // Cardiovasc. Diabetol. — 2014. — Vol. 13. — Article ID: 148. D0I: 10.1186/s12933-014-0148-1.

34. Acheson A. A BDNF autocrine loop in adult sensory neurons prevents cell death / A. Acheson, J.C. Conover, J.P. Fandl [et al.] // Nature. — 1995.— Vol. 374, N6521. - P. 450-453. D01: 10.1038/374450a0.

35. Rauskolb S. Global deprivation of brain-derived neurotrophic factor in the CNS reveals an area-specific requirement for dendritic growth / S. Rauskolb, M. Zagrebelsky, A. Dreznjak [et al.] // J. Neurosci. — 2010. — Vol. 30, N5. — P. 1739-1749. D0I: 10.1523/JNEUROSCI.5100-09.2010.

36. Navaratna D. Decreased cerebrovascular brain-derived neurotrophic factor-mediated neuroprotection in the diabetic brain / D. Navaratna, S.-Z. Guo, K. Hayakawa [et al] // Diabetes. — 2011.—Vol. 60, N6. — P. 1789-1796. D0l: 10.2337/db10-1371.

37. Rani R. Pharmacological investigations on efficacy of Phlorizin a sodium-glucose co-transporter (SGLT) inhibitor in mouse model of intracerebroventricular streptozotocin induced dementia of AD type / R. Rani, A. Kumar, A.S. Jaggi, N. Singh // J. Basic. Clin. Physiol. Pharmacol.— 2021. — D0I: 10.1515/ jbcpp-2020-0330.

38. Hierro-Bujalance C. Empagliflozin reduces vascular damage and cognitive impairment in a mixed murine model of Alzheimer's disease and type 2 diabetes / C. Hierro-Bujalance, C. Infante-Garcia, A. Del Marco [et al.] // Alzheimers Res. Ther. - 2020. — Vol. 12, N1.—Article ID: 40. D0l: 10.1186/s13195-02000607-4.

39. Rizvi S.M.D. Invokana (Canagliflozin) as a dual inhibitor of acetylcholinesterase and sodium glucose co-transporter 2: advancement in Alzheimer's diseasediabetes type 2 linkage via an enzoinformatics study / S.M.D. Rizvi, S. Shakil, D. Biswas [et al.] // CNS Neurol. Disord. Drug Targets. — 2014. — Vol. 13, N3.— P. 447-451. D0I: 10.2174/18715273113126660160.

40. Shaikh S. Forxiga (dapagliflozin): Plausible role in the treatment of diabetes-associated neurological disorders / S. Shaikh, S.M.D. Rizvi, S. Shakil [et al.] // Biotechnol. Appl. Biochem. - 2016. — Vol. 63, N1.—P. 145-150. D0l: 10.1002/bab.1319.

41. Shakil S. Molecular Interaction of Anti-Diabetic Drugs With Acetylcholinesterase and Sodium Glucose Co-Transporter 2/S. Shakil // J. Cell Biochem. — 2017. Vol. 118, N11.—P. 3855-3865. D0I: 10.1002/jcb.26036.

42. Arab H.H. Targeting ROS-Dependent AKT/GSK-3ß/NF-KB and DJ-1/Nrf2 Pathways by Dapagliflozin Attenuates Neuronal Injury and Motor Dysfunction in Rotenone-Induced Parkinson's Disease Rat Model / H.H. Arab, M.M. Safar, N.N. Shahin // ACS Chem. Neurosci.— 2021.— Vol. 12, N4.— P. 689-703. D0l: 10.1021/acschemneuro.0c00722.

43. Arafa N.M.S. Canagliflozin prevents scopolamine-induced memory impairment in rats: Comparison with galantamine hydrobromide action / N.M.S. Arafa, E.H.A Ali, M.K. Hassan // Chem. Biol. Interact._ 2017.—Vol. 277.—P. 195-203. D0I: 10.1016/j.cbi.2017.08.013.

( Колесников Олег Леонидович ( kaf-biol@mail.ru),

Колесникова Алла Алексеевна ( olekol@mail.ru ), Тарабрина Юлия Олеговна ( julikol@mail.ru ).

Журнал «Современная наука: актуальные проблемы теории и практики» 